\title{
Análise de Pesquisas em Economia Compartilhada no Turismo sob a Ótica do Modelo das Três Dicotomias
}

\section{Analysis of Research in the Sharing Economy in Tourism from the Perspective of the Three Dichotomies Model}

\author{
Análisis de Investigaciones en Economía Compartida en el Turismo Bajo la Óptica del \\ Modelo de las Tres Dicotomías
}

\author{
Francis Marcean Resende Barros ${ }^{1}$ \\ Marlusa de Sevilha Gosling ${ }^{2}$
}

\begin{abstract}
Resumo
Os mercados de atividade entre pares estão em crescimento nos serviços de turismo e viagens. O objetivo deste artigo foi desenvolver uma abordagem de pesquisa para estudos futuros que possuam a economia compartilhada como objeto de investigação no contexto do turismo. Realizou-se um levantamento bibliográfico em artigos publicados nos periódicos Tourism Management, Journal of Travel Research e Annals of Tourism Research, classificando-se cada artigo de acordo com o modelo das três dicotomias proposto por Hunt (2010) por meio da técnica de análise de conteúdo. Os resultados métricos mostram que a economia compartilhada é tratada como fenômeno lucrativo (88\%), micro (65\%) e positivo (82\%). Além disso, o estudo apresenta os cinco principais clusters de temas: a) reputação, b) comportamento, c) autenticidade, d) confiança e e) valor. O estudo contribui com pesquisadores, tornando mais clara a compreensão do atual estado da arte em periódicos relevantes e sugerindo uma agenda de pesquisa para direcionar estudos futuros.
\end{abstract}

Palavras-chave: Economia compartilhada. Modelo das Três Dicotomias. Estudo bibliográfico.

\begin{abstract}
Peer activity markets are growing in tourism and travel services. This article aimed to develop a research approach for future studies that have a shared economy as an object of research in the context of tourism. A bibliographic survey was carried out on articles published in the journals Tourism Management, Journal of Travel Research, and Annals of Tourism Research, classifying each article according to the model of the three dichotomies proposed by Hunt (2010) through the technique of analysis of content. The metric results show that the sharing economy is treated as a profitable (88\%), micro (65\%), and positive (82\%) phenomenon. Besides, the study presents the five main clusters of themes: a) reputation, b) behavior, c) authenticity, d) trust, and e) value. The study contributes to researchers, making clearer the understanding of the current state of the art in relevant journals and suggesting a research agenda to guide future studies.
\end{abstract}

Keywords: The sharing economy. Model of the Three Dichotomies. Bibliographic study.

\section{Resumen}

Los mercados de actividad entre pares están en crecimiento en los servicios de turismo y viajes. El objetivo de este trabajo fue desarrollar un enfoque de investigación para futuros estudios que posean la economía compartida como objetivo de investigación en el contexto del turismo. Se realizó una búsqueda bibliográfica en trabajos publicados en los periódicos "Tourism Management", "Journal of Travel Research" y "Annals of Tourism Research", clasificando cada trabajo de acuerdo con el modelo de las tres dicotomías, propuesta por Hunt (2010) por medio de la técnica de análisis de contenido. Los resultados métricos muestran que la economía compartida es tratada como fenómeno lucrativo (88\%), micro (65\%) y positivo (82\%). Además, el trabajo presenta

19 Mestre em Administração pelo Centro de Pesquisas de Pós-Graduação em Administração da Universidade Federal de Minas Gerais (CEPEAD/ UFMG). Doutorando em Administração pelo CEPEAD/UFMG. 
los cinco principales grupos de temas: a) fama, b) comportamiento, c) autenticidad, d) confianza y e) valor. El estudio contribuye con investigadores, aclarando la comprensión del actual estado del arte en periódicos relevantes y sugiriendo una agenda de investigación para direccionar futuros estudios.

Palabras clave: Economía compartida; Modelo de las Tres Dicotomías; Estudio bibliográfico

\section{Introdução}

Os avanços possibilitados pela tecnologia vêm causando rupturas cada vez maiores, transformando estruturas e operações em diversos setores e caucionando a formação de novos mercados, práticas e ofertas (SIGALA, 2018). A tecnologia, portanto, possui papel relevante na criação de novas formas de concatenar a oferta à demanda no rompimento de diversos setores e na aceleração de inovações de negócios, como a economia compartilhada (XIANG, 2017), um fenômeno global que apresenta rápido potencial de crescimento (LUTZ; NEWLANDS, 2018).

A economia compartilhada é uma atividade baseada em pares, ou atividade peer-to-peer, que tem como principal característica a obtenção, a cessão ou o compartilhamento de um produto, que pode ser um bem e/ou um serviço, entre pares (HAMARI; SJÖKLINT; UKKONEN, 2016), significando uma alternativa à propriedade particular (BELK, 2009). Embora o compartilhamento não seja um fenômeno estritamente atual, ele era limitado a familiares e amigos (GUNTER, 2018). Assim, a economia compartilhada se diferencia pela ampliação das relações de compartilhamento, tendo em vista que os bens e/ou serviços passam a ser compartilhados entre pessoas desconhecidas entre si (HAWLITSCHEK; TEUBNER; WEINHARDT, 2016).

Estabelecida além dos limites de uma tendência temporária e frágil (BELK, 2014b), a economia compartilhada é um assunto relevante na atualidade, cuja força é capaz de gerar impactos no desenvolvimento sustentável e construir comunidades mais fortes (BOTSMAN; ROGERS, 2011). Além disso, os negócios que possuem um modelo peer-topeer são considerados prioridades para pesquisas (CHENG, 2016), denotando rápido crescimento, especialmente em serviços de turismo e viagens (PIZAM, 2014).

Mediante a relevância do fenômeno, torna-se igualmente relevante analisar sua natureza. O modelo das três dicotomias (HUNT, 2010) aventa a classificação de todos os fenômenos, tópicos e questões de marketing conforme as dicotomias (1) de finalidade: lucrativo/não-lucrativo; (2) de agregação: micro/macro, e (3) de perspectiva: positiva/ normativa, gerando um total de $2 \times 2 \times 2=8$ células ou classes de enquadramento. Considerando essa diversidade de classificações, procura-se entender a posição de fenômenos no referido modelo e, assim, identificar abordagens que podem ser tomadas por estudos futuros.

Dado o contexto, o objetivo geral deste artigo foi conceber uma abordagem de pesquisa para estudos sobre economia compartilhada no turismo. Para tanto, buscou-se analisar as publicações em periódicos relevantes da área por meio do modelo das três dicotomias, identificar clusters de temas nos artigos pesquisados e traçar uma agenda de pesquisa para conduzir estudos futuros na temática.

\section{Economia Compartilhada}

Em razão da novidade trazida pela economia compartilhada, ela é frequentemente alvo de ambiguidade sobre o seu significado (FRENKEN, SCHOR, 2017). Por isso, ela é frequentemente aplicada de maneira equivocada a modelos de eficiência que apenas conectam a oferta à demanda, como aplicativos de serviços de entrega, que não apresentam traço algum de colaboração entre os envolvidos (BOTSMAN, 2015).

A economia compartilhada tem base em um fenômeno mais amplo, o compartilhamento. Com isso, é importante diferenciar os conceitos que estão envolvidos no âmbito do compartilhamento. Na Figura 1 estão sintetizadas as diferenças principais entre os fenômenos compartilhamento, consumo colaborativo e outras modalidades mais tradicionais de transação (aluguel e compra) de acordo com Benoit et al. (2017).

O aluguel e a compra envolvem relações diádicas (BENOIT et al., 2017), sendo que, na compra, a transação se baseia na transferência da posse (BENOIT et al., 2017; HAASE; KLEINALTENKAMP, 2011), enquanto o aluguel envolve um acesso a um bem ou a um serviço (BENOIT et al., 2017), geralmente ocorrendo em um período mais longo se comparado a outras formas de acesso (MOELLER; WITTKOWSKI, 2010).

O consumo colaborativo envolve, essencialmente, três atores: um provedor de serviços, um consumidor que procura acessá-lo e uma plataforma que realiza a mediação dessa troca. A troca no consumo colaborativo implica que os clientes obterão acesso a recursos, tangíveis ou não (WITTKOWSKI; MOELLER; WIRTZ, 2013), envolvendo, necessariamente, uma compensação monetária (HAASE; KLEINALTENKAMP, 2011). Contudo essa troca não envolve a propriedade em sentido estrito. A propriedade envolvida no consumo colaborativo é temporária, isto é, um fornecedor que possui um recurso o concede a outros indivíduos, como ocorre com um ator que possui um veículo e o cede a outros que precisam de transporte temporário (HAASE; KLEINALTENKAMP, 2011). 
Figura 1. Consumo colaborativo e outros fenômenos

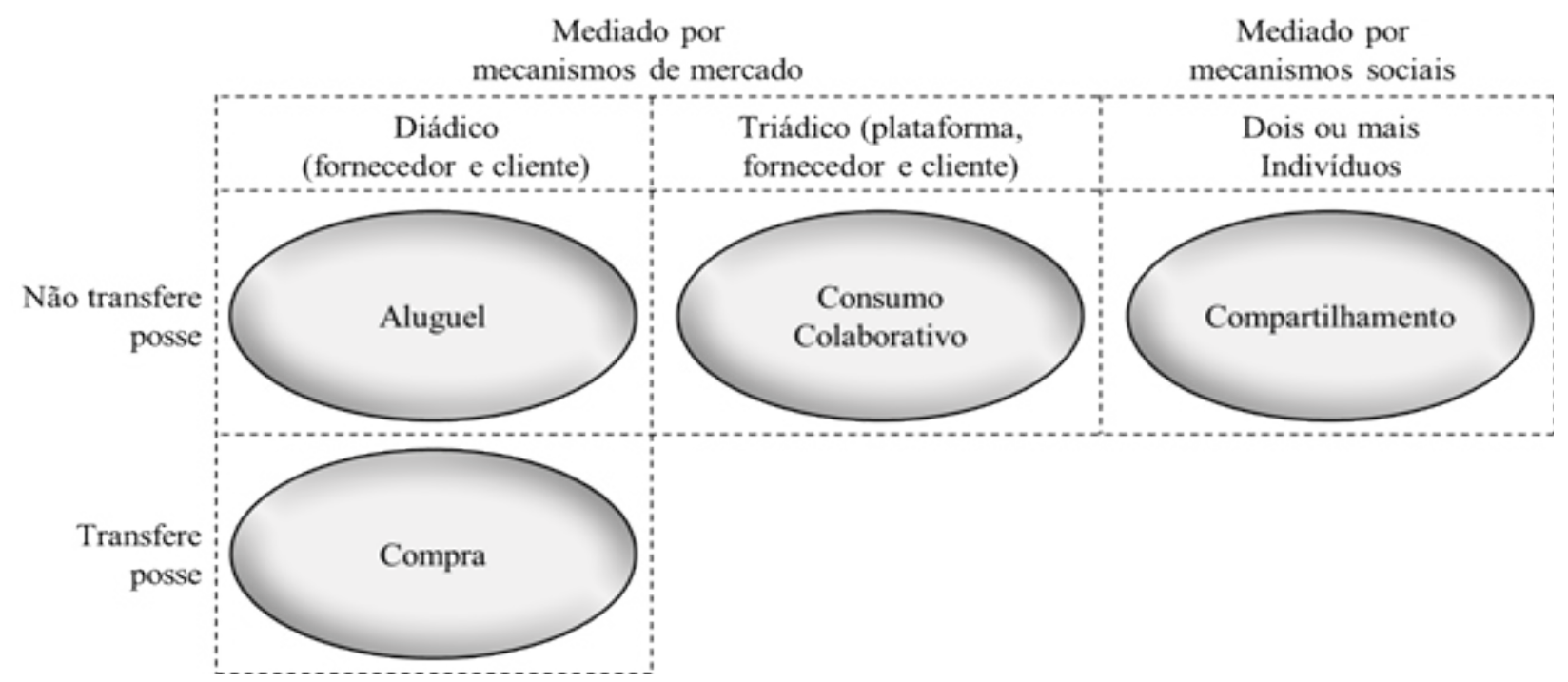

Fonte: Adaptado de Benoit et al. (2017).

O compartilhamento, por sua vez, é mediado por mecanismos sociais (HILL; WELLMAN, 2012), ocorrendo dentro de grupos que são conectados socialmente, como a família ou os amigos próximos (BELK, 2009). Ele não envolve tríades, necessariamente. No entanto também pode ocorrer em um triângulo de atores, como ocorre com o consumo colaborativo e, ainda assim, normalmente, acontece entre os indivíduos, de maneira que nenhuma contrapartida monetária está envolvida (BENOIT et al., 2017).

Diante do exposto, considera-se que a economia compartilhada pode ser entendida como um conjunto das práticas de compartilhamento e de consumo colaborativo que se realizam no âmbito de plataformas digitais. Essa restrição [somente em plataformas digitais] se dá em virtude de a economia compartilhada ter nascido por meio dos aplicativos e plataformas criados com o advento da Web 2.0 (BELK, 2014b).

\section{Modelo das Três Dicotomias}

Hunt (2010) considera que é possível classificar os fenômenos de marketing por meio do modelo das três dicotomias dada a sua estrutura analítica. Esta seção discorre sobre as dicotomias, enfatizando suas características e as manifestações do fenômeno da economia compartilhada em seu contexto.

\subsection{Lucrativo/não lucrativo}

Na dicotomia lucrativo/não lucrativo, o lucrativo está relacionado aos estudos que aderem à perspectiva de orientação para lucros, abarcando os estudos em organizações e/ou demais entidades que incluem a geração de lucro em seus objetivos. Já o não lucrativo trata de estudos na perspectiva de organizações e entidades em que o lucro não é tomado como objetivo (HUNT, 2010).

No contexto da economia compartilhada, há um debate sobre duas formas de compartilhamento, chamadas de "compartilhamento verdadeiro" e "pseudocompartilhamento". Não é a característica do que é compartilhado que determina sua natureza, mas as intenções dos envolvidos. O compartilhamento verdadeiro está mais ligado a uma atitude altruísta, que envolve também aspectos emotivos de criação de relações e de comunidade. Por outro lado, o pseudocompartilhamento pode ser motivado por dinheiro, egoísmo, expectativas de reciprocidade e falta de senso de comunidade, tratando-se de uma relação comercial, isto é, da troca de produtos por dinheiro. É uma relação comercial camuflada em um compartilhamento, servindo a motivos utilitaristas, como obtenção de lucro (BELK, 2014a).

Analisando a dicotomia e as formas de compartilhamento, é possível realizar uma analogia entre (1) o lucrativo e o pseudocompartilhamento e (2) o não lucrativo e o compartilhamento verdadeiro. Belk (2014b) faz menção ao consumo colaborativo (às vezes, tomado como sinônimo da economia compartilhada) como exemplo do pseudocompartilhamento, alegando que não se trata de um compartilhamento verdadeiro por enfatizar uma relação comercial. As empresas Airbnb e Uber são exemplos de plataformas lucrativas, enquanto o Couchsurfing representa uma plataforma de hospedagem compartilhada que não prevê dispêndio financeiro do usuário. 


\subsection{Micro/macro}

A dicotomia micro/macro é a mais ambígua das três. A diferenciação se dá, de forma análoga à distinção entre microeconomia e macroeconomia, levando em conta a agregação. O micro (ou micromarketing) diz respeito às atividades de marketing que ocorrem em unidades individuais, como empresas, consumidores ou famílias. O macro (ou macromarketing) está voltado para um nível ampliado de agregação, ou seja, envolve sistemas de marketing ou grupos de consumidores (HUNT, 2010).

Levando em consideração o critério de agregação, tópicos como a responsabilidade social do marketing não seriam suficientes para sua classificação no âmbito macro, embora a maioria dos profissionais da área do marketing anseie por classificar tópicos relativos às responsabilidades sociais no plano macro. Em aproximações, como a especificação do que é micro e do que é macro em termos de "internalidades" e "externalidades", em que o micro é relacionado às atividades internas de empresas (internalidades) e o macro aos interesses da sociedade (externalidades), o macro poderia incorporar as responsabilidades sociais. Todavia tal especificação não abrangeria tópicos que fogem aos interesses da sociedade, como aspectos legais, marketing comparativo e relacionamento em canais de distribuição, tornando o critério de marketing social insuficiente para classificação do macromarketing (HUNT, 2010).

O macromarketing é, portanto, tido como um construto multidimensional. Assim, uma especificação mais completa inclui os seguintes critérios: estudo (1) de sistemas de marketing, em que a agregação absorve tópicos como marketing comparativo, relacionamentos nos canais de distribuição e estrutura de marketing de relacionamento; (2) do impacto dos sistemas de marketing na sociedade, que abarca as responsabilidades sociais e o marketing como intensificador do desenvolvimento econômico; e (3) do impacto da sociedade em sistemas de marketing, como aspectos legais e consequências para o marketing sob o ponto de vista dos valores políticos e sociais (HUNT, 2010).

Estudos apontam que a economia compartilhada tem caráter disruptivo em termos de canais (GUTTENTAG, 2015) e em termos estruturais (LAURELL; SANDSTRÖM, 2016), impacta na sociedade (HABIBI; DAVIDSON; LAROCHE, 2017; RAHDARI; SEPASI; MORADI, 2016; WU; ZHI, 2016), na sustentabilidade (HABIBI; DAVIDSON; LAROCHE, 2017; WU; ZHI, 2016) e sofre pressão política (MAIR; REISCHAUER, 2017) e de regulamentação (HOU, 2018). No entanto, cabe a análise de objetos de estudo para sua classificação nessa dicotomia, considerando o critério de agregação pontuado por Hunt (2010).

\subsection{Positivo/normativo}

Hunt (2010) faz a diferenciação entre marketing positivo e marketing normativo. O marketing positivo é o que tem como finalidade a tentativa de descrição, explicação, previsão e compreensão das atividades de marketing e de fenômenos realmente existentes, ou seja, examina o que efetivamente é [a atividade ou fenômeno de marketing]. Por outro lado, o marketing normativo adota uma perspectiva de caráter prescritivo, ou seja, está imbuído em prescrever ou recomendar quais ações as organizações e indivíduos deveriam executar ou quais formatos os sistemas de marketing deveriam ter, dessa forma, a perspectiva normativa examina o que as organizações e indivíduos deveriam ser e o que deveriam fazer (HUNT, 2010).

Muñoz e Cohen (2017) afirmam que as pesquisas sobre economia compartilhada são, em sua maioria, de natureza normativa. Acquier, Daudigeos e Pinkse (2017) argumentam que a economia compartilhada possui base e caráter normativos, cujo debate é interminável e discorre sobre o que ela deveria ser ou não consegue ser. Contudo os mesmos autores pontuam que encontrar um caminho entre os extremos (normativo/positivo) é uma questãochave, balizando visões relativistas ou restritas. Assim, não se torna clara a sua classificação em termos de evidências teóricas.

\section{Procedimentos Metodológicos}

Quanto aos objetivos, este estudo é exploratório. A pesquisa exploratória tem, como uma das finalidades, a formulação de perguntas para estudos posteriores, sendo executada, habitualmente, por meio de estudo bibliográfico e documental (GIL, 2008). Quanto à natureza, é um artigo teórico (ALAVl; CARLSON, 1992), pois se baseia principalmente em ideias e estruturas, contendo observações que possuem papel secundário na condução do estudo.

Os dados utilizados para a condução do estudo são secundários. Na maioria das vezes, a obtenção de dados secundários é mais rápida. Além disso, são mais acessíveis em relação aos dados primários (RÉVILLION, 2015). A abordagem é qualitativa, uma vez que se pretendeu analisar os dados por meio de uma perspectiva interpretativa. A subseção seguinte discute a análise dos dados com maior profundidade.

Quanto à seleção dos dados, foram considerados os artigos dos três periódicos mais influentes na categoria "Gestão de turismo, lazer e hospitalidade" do SCImago Journal Rank Indicator'. Os periódicos estão localizados no primeiro quartil do ranking. São eles: Tourism Management, Journal of Travel Research e Annals of Tourism Research. Nacionalmente, todos são classificados como Qualis A1 na Plataforma Sucupira/Capes.

1 Disponível em: $<$ https://www.scimagojr.com/journalrank.php?category=1409\&year=2017> Acesso em 26 ago. 2018. 
Dado que os periódicos são publicados em língua inglesa, realizou-se uma pesquisa pelo termo "sharing economy". Obteve-se o total de 20 artigos no Tourism Management, 7 artigos no Journal of Travel Research e 6 artigos no Annals of Tourism Research, totalizando 33 artigos. Nessa busca, consideraram-se artigos denominados por "research articles", que envolvem estudos com utilização de dados primários².

Foi realizada uma revisão bibliográfica. Esse tipo de pesquisa é desenvolvida a partir de material já construído em livros ou artigos científicos, geralmente envolvendo estudos exploratórios realizados por meio da técnica de análise de conteúdo (GIL, 2008). A técnica de análise de conteúdo (BARDIN, 2011) foi utilizada em uma abordagem interpretativa, ou seja, os autores da presente pesquisa, a partir da leitura, fizeram a cessão do significado ao conteúdo.

Na pré-análise, realizou-se a seleção dos documentos (abordada na subseção anterior), escolhendo os artigos com pesquisas empíricas para constituir o corpus de análise. Na exploração do material, após leitura flutuante, consideraram-se 9 artigos da Tourism Management, 5 da Journal of Travel Research e 3 da Annals of Tourism Research por estarem enquadrados na temática investigada. Para a categorização, tomou-se cada polo das três dicotomias do modelo proposto por Hunt (2010). Assim, no tratamento dos resultados e interpretação, buscaram-se evidências que permitiram realizar a classificação dos artigos nas categorias identificadas por meio da justaposição dos resultados ao referencial teórico.

\section{Resultados e Discussão}

O Quadro 1 descreve o título e objetivo geral dos artigos por periódico analisado. Optou-se por preservar o título original dos artigos e traduzir apenas o objetivo geral. Essa sistematização permitiu identificar os clusters de temas, bem como gerar os primeiros insights para classificação dos artigos de acordo com o modelo proposto.

Quadro 1: Título e objetivo geral dos artigos

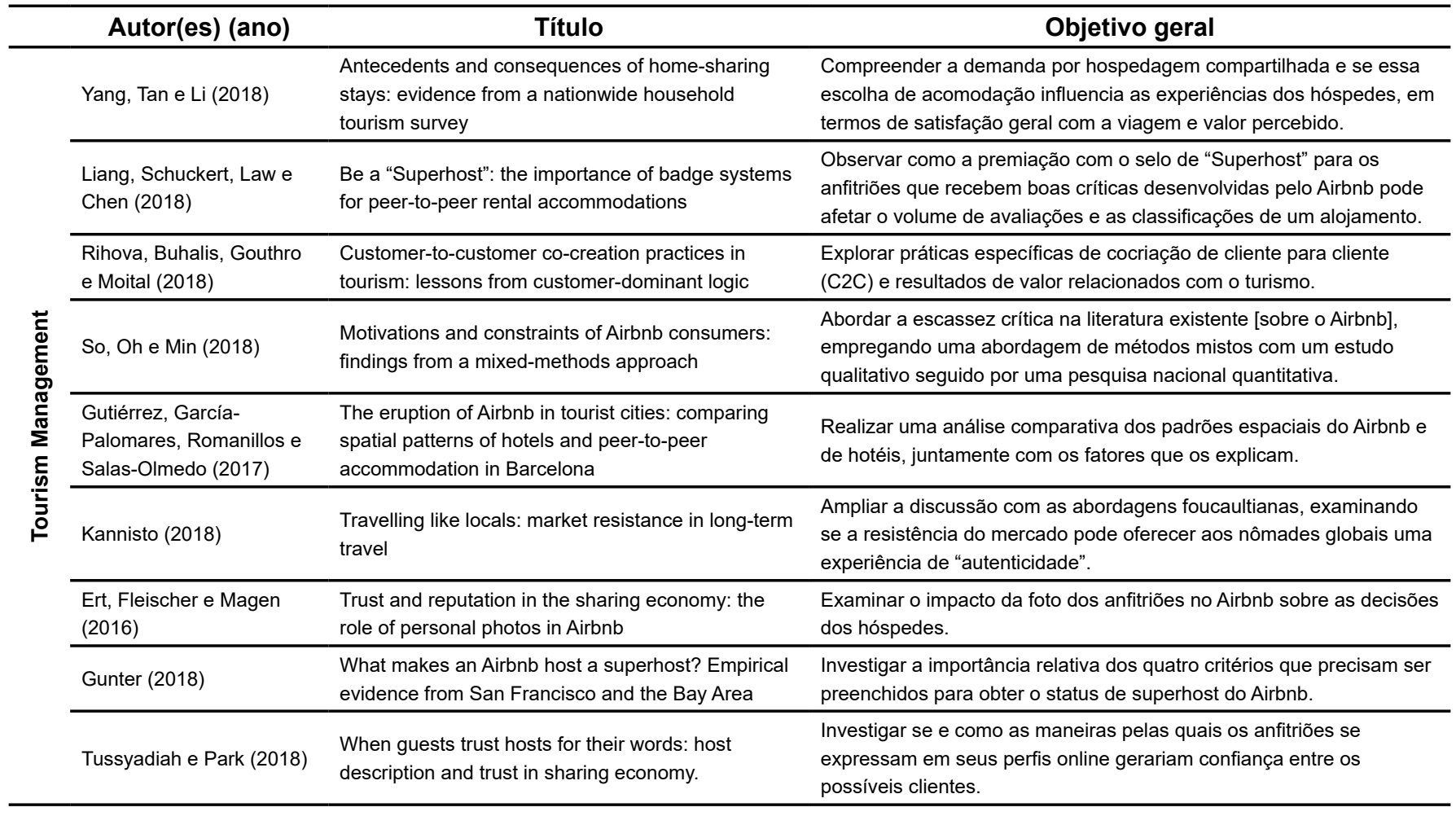

Fonte: Elaborado pelos pesquisadores (2018).

2 Disponível em: $<$ https://apus.libanswers.com/faq/2324> Acesso em 26 ago. 2018. 
Quadro 1: Título e objetivo geral dos artigos (continuação)

\begin{tabular}{|c|c|c|c|}
\hline \multirow{5}{*}{ 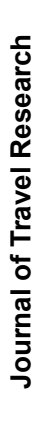 } & $\begin{array}{l}\text { Tussyadiah e Pesonen } \\
\text { (2016) }\end{array}$ & $\begin{array}{l}\text { Impacts of peer-to-peer accommodation use on } \\
\text { travel patterns }\end{array}$ & $\begin{array}{l}\text { Identificar como o uso de acomodação compartilhada leva a } \\
\text { mudanças no comportamento dos viajantes. }\end{array}$ \\
\hline & $\begin{array}{l}\text { Hajibaba, Karlsson e } \\
\text { Dolnicar (2017) }\end{array}$ & $\begin{array}{l}\text { Residents open their homes to tourists when } \\
\text { disaster strikes }\end{array}$ & $\begin{array}{l}\text { Investigar o potencial do envolvimento dos residentes na recuperação } \\
\text { dos destinos turísticos após um desastre, bem como durante a } \\
\text { emergência imediata do desastre. }\end{array}$ \\
\hline & $\begin{array}{l}\text { Guttentag, Smith, } \\
\text { Potwarka e Havitz (2018) }\end{array}$ & $\begin{array}{l}\text { Why tourists choose Airbnb: a motivation-based } \\
\text { segmentation study }\end{array}$ & $\begin{array}{l}\text { Investigar as motivações dos turistas para usar o Airbnb e segmentá- } \\
\text { los. }\end{array}$ \\
\hline & Abrate e Viglia (2019) & $\begin{array}{l}\text { Personal or product reputation? Optimizing } \\
\text { revenues in the sharing economy }\end{array}$ & $\begin{array}{l}\text { Examinar como os vendedores precisam melhorar sua reputação } \\
\text { pessoal para otimizar as receitas. }\end{array}$ \\
\hline & $\begin{array}{l}\text { Garau-Vadell, Gutiérrez- } \\
\text { Taño e Díaz-Armas (2019) }\end{array}$ & $\begin{array}{l}\text { Residents' support for } \mathrm{p} 2 \mathrm{p} \text { accommodation in mass } \\
\text { tourism destinations }\end{array}$ & $\begin{array}{l}\text { Fornecer um modelo explicativo para mostrar como a percepção dos } \\
\text { impactos da atividade influencia as atitudes e o apoio dos residentes. }\end{array}$ \\
\hline \multirow{3}{*}{ 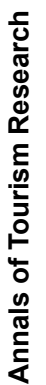 } & Ye, Xiao e Zhou (2018) & $\begin{array}{l}\text { Commodification and perceived authenticity in } \\
\text { commercial homes }\end{array}$ & $\begin{array}{l}\text { Examinar a interação da mercantilização e autenticidade percebida de } \\
\text { casas comerciais em áreas rurais. }\end{array}$ \\
\hline & $\begin{array}{l}\text { Karlsson, Kemperman e } \\
\text { Dolnicar (2017) }\end{array}$ & May i sleep in your bed? Getting permission to book & Investigar o requisito de solicitar permissão para se hospedar. \\
\hline & Molz (2013) & $\begin{array}{l}\text { Social networking technologies and the moral } \\
\text { economy of alternative tourism: the case of } \\
\text { couchsurfing.org }\end{array}$ & $\begin{array}{l}\text { Examinar o papel das tecnologias de redes sociais na economia } \\
\text { moral do turismo alternativo. }\end{array}$ \\
\hline
\end{tabular}

Fonte: Elaborado pelos pesquisadores (2018).

Para identificação de clusters de temas, foram considerados o objeto central dos estudos e os seus enfoques. É importante salientar que os artigos selecionados já são, por si só, partes de um cluster que é a economia compartilhada no contexto do turismo. Assim, este esforço em identificar temas tem a intenção de descrever o que está sendo discutido.

Em termos de objeto de pesquisa, o tema de hospedagem compartilhada apareceu em 15 dos 17 artigos. Sendo assim, optou-se por tomar como temas os enfoques utilizados nas pesquisas. A Figura 2 ilustra os temas abordados. $O$ tema reputação foi o mais tratado em cerca de $24 \%$ estudos. Liang et al. (2018) e Gunter (2018) investigaram os superhosts (ou super anfitriões) do Airbnb. Ert, Fleischer e Magen (2016) investigaram o impacto da foto dos anfitriões na escolha do hóspede, já Abrate e Viglia (2019) examinaram como os hosts podiam aumentar a receita melhorando a reputação pessoal.

Figura 2. Clusters de temas (por enfoque)

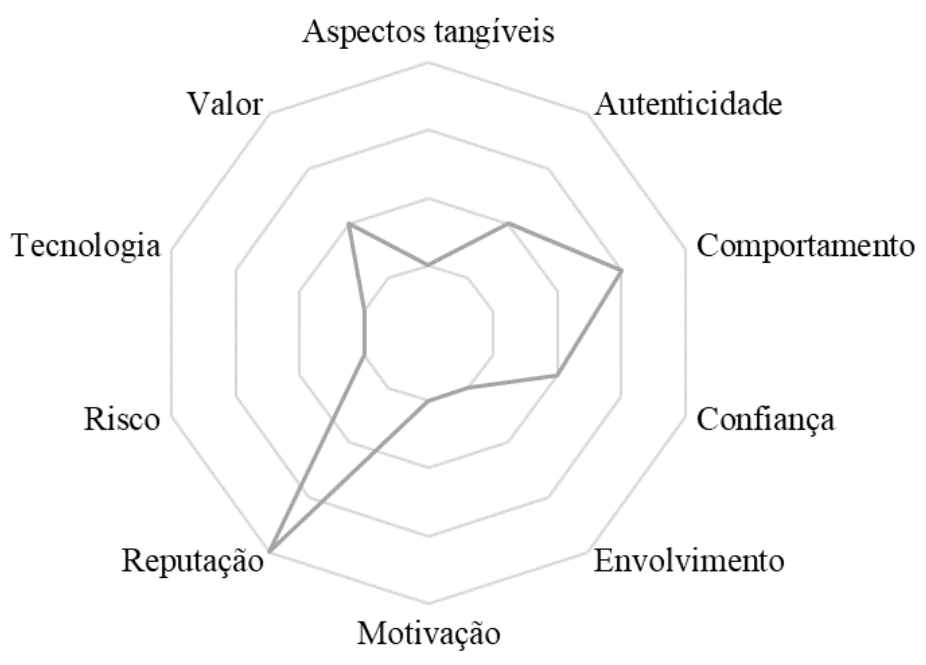

Fonte: Elaborado pelos pesquisadores (2018).

Os demais temas foram comportamento (18\%), valor, autenticidade e confiança (12\% cada). Tussyadiah e Pesonen (2016) investigaram a mudança de comportamento decorrente do uso de hospedagem compartilhada. Rihova et al. (2018) exploraram o valor resultante de práticas de cocriação. Ye, Xiao e Zhou (2018) examinaram a autenticidade percebida de casas comerciais em zonas rurais. Quanto à confiança, Tussyadiah e Park (2018) investigaram a geração de confiança pelos anfitriões por meio de seus perfis on-line. 


\subsection{Análise e discussão dos resultados}

Antes de se proceder à análise e discussão dos resultados, é importante salientar as colocações pontuadas na seção 2 deste trabalho. Os achados deste trabalho são voltados ao âmbito da economia compartilhada, que é um entre os outros diversos mecanismos de transação entre indivíduos. É importante, dessa forma, não confundir a economia compartilhada com o consumo colaborativo (que trata de um ponto específico da economia compartilhada) ou com o compartilhamento puro (que é um fenômeno muito mais antigo).

A Tabela 1 resume a classificação dos artigos por periódico em um panorama geral. Os três periódicos demonstraram que o fenômeno é tratado, em maioria, como lucrativo (88\%), micro (65\%) e positivo (82\%).

Tabela 1. Classificação dos artigos TM

\begin{tabular}{|l|c|c|c|c|c|c|}
\hline & Lucrativo & Não lucrativo & Micro & Macro & Normativo & Positivo \\
\hline Tourism Management & $89 \%$ & $11 \%$ & $67 \%$ & $33 \%$ & $22 \%$ & $78 \%$ \\
\hline Journal of Travel Research & $100 \%$ & $0 \%$ & $60 \%$ & $40 \%$ & $0 \%$ & $100 \%$ \\
\hline Annals of Tourism Research & $67 \%$ & $33 \%$ & $67 \%$ & $33 \%$ & $33 \%$ & $67 \%$ \\
\hline Geral & $88 \%$ & $12 \%$ & $65 \%$ & $35 \%$ & $18 \%$ & $82 \%$ \\
\hline
\end{tabular}

Fonte: Elaborado pelos pesquisadores (2018).

Observa-se que os estudos empíricos estão mais voltados para o aspecto lucrativo da economia compartilhada, sendo essa dicotomia a que representou maior amplitude entre os percentuais nos três periódicos. É possível verificar isso a partir do volume de estudos que utilizaram o Airbnb como objeto de investigação, justificando sempre pela sua capacidade comercial. No Journal of Travel Research essa situação ficou evidente, dado que todos os artigos possuem objetos de estudo cujas finalidades são lucrativas.

Para Opperman (2017), as plataformas de economia compartilhada pautadas em trocas comerciais (consumo colaborativo) possuem mais sucesso. Bucher, Fieseler e Lutz (2016) retrataram que o incentivo monetário é uma condição necessária para o compartilhamento. Além disso, estima-se que as receitas de empresas de economia compartilhada podem alcançar mais de US\$ 335 bilhões até 2025 (PRICEWATERHOUSE INTERNATIONAL LTD., 2015).

Benoit et al. (2017) reforçam que o consumo colaborativo é mediado por mecanismos de mercado, o que o diferencia de fenômenos relacionados (como compartilhamento ou serviços baseados em acesso, compra ou aluguel). Souza e Lemos (2016) exemplificam por meio de empresas, como a Uber e o AirBnb, que viabilizam locomoção e acomodação em cidades sem possuírem frotas ou quartos próprios, atuando de forma intermediária entre os polos interessados.

No entanto cumpre esclarecer que isso reflete o pseudocompartilhamento (BELK, 2014a), representando uma tendência em se comercializar o compartilhamento que, outrora, era uma atividade voltada ao compartilhamento sem transação comercial, cujo exemplo é o Couchsurfing, que esteve presente em apenas um artigo analisado (MOLZ, 2013), sendo também o mais antigo.

Quanto à agregação, a maior parte dos artigos considerou o aspecto micro da economia compartilhada, sendo essa dicotomia a que apresentou menor amplitude entre os seus polos. Em muitos casos, analisaram-se estritamente as interações entre cliente e provedor de serviço. Em outros, no entanto, algumas variáveis relacionadas à externalidades foram consideradas e discutidas.

So, Oh e Min (2018) foram assertivos ao considerar a agregação do fenômeno, pois utilizaram uma abordagem multimétodos para abordar a escassez crítica na literatura sobre o Airbnb, provendo contribuições enquanto sistema de marketing. Hajibaba, Karlsson e Dolnicar (2017) também abordaram o fenômeno além da esfera do micromarketing, versando sobre um senso de comunidade entre usuários, refletindo, além da abordagem sistêmica do fenômeno, sobre as relações recíprocas entre sociedade e plataformas de economia compartilhada.

É importante salientar que os artigos trazem recortes que, quase automaticamente, inibem sua capacidade de tratar o assunto de maneira mais agregada, todavia, ainda que o fenômeno como um todo possua base que atenda aos critérios de agregação colocados por Hunt (2010), as evidências empíricas mostram, em maioria, que o assunto tem sido tratado de maneira menos agregada. Dessa forma, para atender o objetivo de traçar uma agenda de pesquisa, o fenômeno será tomado como micro.

Em relação à perspectiva, a maioria dos trabalhos partiu de problemas ou objetivos positivos, ou seja, com finalidade de descrição e/ou explicação. Com a segunda maior amplitude, a primazia do positivo é expressa pelos artigos do Journal of Travel Research, em que todos apresentaram uma perspectiva positiva na pesquisa realizada.

A economia compartilhada apresenta base e caráter normativos, cujo debate é interminável e discorre sobre o que ela deveria ser ou não consegue ser (ACQUIER; DAUDIGEOS; PINKSE, 2017). Kannisto (2018), por exemplo, seguiu a perspectiva de que as experiências autênticas do turismo deveriam ser buscadas além do consumo, ainda que seja improvável escapar totalmente de um mercado.

Por outro lado, a maioria dos artigos já trata o tema como ele é, não como deveria ou poderia ser, mostrando 
que aspectos normativos sobre o fenômeno podem ter se dissipado ao passo em que ele deixou de ser incipiente. Logo, da mesma forma em que se considerou a agregação micro com base nas evidências empíricas, considera-se, também, o fenômeno como positivo.

\section{Considerações Finais}

As atividades de empresas atuantes na economia compartilhada são, em sua maioria, ligadas a fins lucrativos. Neste ensaio, foi possível estabelecer uma analogia entre o lucrativo e o pseudocompartilhamento, que apresenta primazia perante o compartilhamento verdadeiro, sustentada pelo advento do capitalismo e pelo próprio comportamento das pessoas orientado pelo lucro ou, mais especificamente, pelo dinheiro. As crises, as mudanças econômicas e o desemprego também justificam parte da preferência por plataformas que têm o lucro como finalidade, pois a economia compartilhada, nesse aspecto, representa uma forma de aumentar a rentabilidade por meio do uso de ativos subutilizados.

Quanto à dicotomia micro/macro, a economia compartilhada atende aos critérios colocados por Hunt (2010) para pertencer ao âmbito macro, contudo os estudos analisados mostram outro lado. A maioria das pesquisas abordou o fenômeno de forma limitada, não o tratando como sistema de marketing que tem relações recíprocas com a sociedade.

No que concerne às perspectivas positiva e normativa, embora a base da economia compartilhada seja mais normativa, os estudos sobre o fenômeno já apresentam finalidade de descrição e explicação.

Analisando, portanto, a posição da economia compartilhada nas três dicotomias, chegou-se ao resultado de que esse fenômeno é enquadrado na célula lucrativa, micro e positiva do modelo. Como limitação, tem-se que a utilização do modelo das três dicotomias para a proposta da agenda de pesquisa, dado seu caráter classificatório, excluiu alguns modelos de negócio que operam dentro do fenômeno, como as plataformas não lucrativas. Portanto, sugere-se que tais plataformas também possam ser analisadas e classificadas em uma abordagem de pesquisa.

\subsection{Implicações técnicas e limitações}

Este trabalho traz duas principais contribuições técnicas para os estudos sobre economia compartilhada. A primeira se refere à melhoria na compreensão e na abordagem do fenômeno, mostrando suas facetas e levantando os principais pontos à luz de um modelo relevante. Outro aspecto é a classificação de um fenômeno considerando sua finalidade (lucrativa/ não lucrativa), sua abrangência (micro/macro) e sua perspectiva (positiva/normativa), que chama atenção para novos questionamentos e inquietações para o fomento de pesquisas, cujas possibilidades são discutidas na subseção a seguir.

Por outro lado, é importante salientar que essa pesquisa focou estritamente na economia compartilhada. Dessa forma, temas como o consumo colaborativo, especificamente, ou o compartilhamento puro, não foram aprofundados, o que representa uma limitação desse estudo. Assim, além das propostas para estudos que serão apresentadas na subseção 6.2, sugere-se uma réplica deste estudo que abarque mais fenômenos derivados do compartilhamento, inclusive o próprio compartilhamento.

\subsection{Agenda de pesquisa}

As plataformas de compartilhamento on-line trouxeram novos questionamentos. Elas vêm em formas variadas e operam em vários setores, destacando distintos efeitos sobre a economia, meio ambiente e sociedade. Nesse contexto, há necessidade de pesquisas para entender o funcionamento dessas plataformas.

Para fenômenos de marketing enquadrados na célula lucrativo/micro/positivo, Hunt (2010, p. 12) propõe um esquema de:

\footnotetext{
"problemas, questões, teorias e pesquisas sobre: comportamento individual do consumidor; como as empresas determinam os preços; como as empresas determinam os produtos; como as empresas determinam a promoção; como as empresas determinam os canais de distribuição; e estudos de caso de práticas de marketing".
}

Assim, com base nessa abordagem, finaliza-se esse ensaio com uma proposta para realização de estudos futuros pautados nas temáticas de mix de marketing e práticas de marketing.

Mix de marketing: quais critérios são utilizados pelos prestadores de serviço das plataformas peer-to-peer para precificação do produto? Como são desenvolvidos os produtos dessas empresas? Quais estratégias de promoção são mais eficazes? Como são gerenciados os canais de distribuição em termos da relação empresa x prestador de serviço x usuário?

Práticas de marketing: como as empresas de economia compartilhada realizam as atividades de marketing? Qual abordagem é utilizada pelos programas de marketing dessas empresas? Quais práticas são realizadas pelos prestadores de serviço das plataformas peer-to-peer? 


\section{Referências}

ABRATE, G.; VIGLIA, G. Personal or product reputation? Optimizing revenues in the sharing economy. Journal of Travel Research, [s. I], v. 58, n. 1, p. 136-148, 2019.

ACQUIER, A.; DAUDIGEOS, T.; PINKSE, J. Promises and paradoxes of the sharing economy: an organizing framework. Technological Forecasting and Social Change, [s. I], v. 125, p. 1-10, 2017.

ALAVI, M.; CARLSON, P. A review of MIS research and disciplinary development. Journal of Management Information Systems, [s. I], v. 8, n. 4, p. 45-62, 1992.

BARDIN, L. Análise de conteúdo. São Paulo: Edições 70, 2011.

BELK, R. Sharing versus pseudo-sharing in Web 2.0. The Anthropologist, [s. I], v. 18, n. 1, p. 7 23, $2014 a$.

BELK, R. Sharing. Journal of consumer research, [s. I], v. 36, n. 5, p. 715-734, 2009.

BELK, R. You are what you can access: sharing and collaborative consumption online. Journal of Business Research, [s. I], v. 67, n. 8, p. 1595-1600, 2014b.

BENOIT, S. et al. A triadic framework for collaborative consumption (CC): motives, activities and resources \& capabilities of actors. Journal of Business Research, [s. I], v. 79, p. 219-227, 2017.

BOTSMAN, R.; ROGERS, R. What's mine is yours: how collaborative consumption is changing the way we live. Reino Unido: HarperCollins UK, 2011.

BOTSMAN, R. Defining the sharing economy: What is collaborative consumption-and What Isn't? Disponível em: $<$ https://www.fastcompany.com/3046119/defining-the-sharing-economy-what-is-collaborative-consumption-and-whatisnt> (2015). Acesso em 11 out. 2018.

BUCHER, E.; FIESELER, C.; LUTZ, C. What's mine is yours (for a nominal fee)-Exploring the spectrum of utilitarian to altruistic motives for Internet-mediated sharing. Computers in Human Behavior, [s. I], v. 62, p. 316-326, 2016.

CHENG, M. Current sharing economy media discourse in tourism. Annals of Tourism Research, v. 60, n. C, p. 111-114, 2016.

ERT, E.; FLEISCHER, A.; MAGEN, N. Trust and reputation in the sharing economy: the role of personal photos in Airbnb. Tourism Management, [s. I], v. 55, p. 62-73, Aug. 2016.

FRENKEN, K.; SCHOR, J. Putting the sharing economy into perspective. Environmental Innovation and Societal Transitions, [s. I], v. 23, p. 3-10, Jun. 2017.

GARAU-VADELL, J.; GUTIÉRREZ-TAÑO, D.; DÍAZ-ARMAS, R. Residents' support for P2P accommodation in mass tourism destinations. Journal of Travel Research, [s. I], v.58, n.4, p. 549-565, 2019.

GIL, A. Métodos e técnicas de pesquisa social. 6.ed. São Paulo: Editora Atlas SA, 2008.

GUNTER, U. What makes an airbnb host a superhost? Empirical evidence from San Francisco and the Bay Area. Tourism Management, [s. I], v. 66, p. 26-37, 2018.

GUTIÉRREZ, J. et al. The eruption of Airbnb in tourist cities: comparing spatial patterns of hotels and peer-to-peer accommodation in Barcelona. Tourism Management, [s. I], v. 62, p. 278-291, 2017.

GUTTENTAG, D. Airbnb: disruptive innovation and the rise of an informal tourism accommodation sector. Current issues in Tourism, [s. I], v. 18, n. 12, p. 1192-1217, 2015.

GUTTENTAG, D. et al. Why tourists choose Airbnb: a motivation-based segmentation study. Journal of Travel Research, [s. I], v. 57, n. 3, p. 342-359, 2018.

HAASE, M.; KLEINALTENKAMP, M. Property rights design and market process: implications for market theory, 
marketing theory, and SD Logic. Journal of Macromarketing, [s. ], v. 31, n. 2, p. 148-159, 2011.

HABIBI, M.; DAVIDSON, A.; LAROCHE, M. What managers should know about the sharing economy. Business Horizons, [s. I], v. 60, n. 1, p. 113-121, 2017.

HAJIBABA, H.; KARLSSON, L.; DOLNICAR, S. Residents open their homes to tourists when disaster strikes. Journal of Travel Research, [s. I], v. 56, n. 8, p. 1065-1078, 2017.

HAMARI, J.; SJÖKLINT, M.; UKKONEN, A. The sharing economy: Why people participate in collaborative consumption. Journal of the Association for Information Science and Technology, [s. I], v. 67, n. 9, p. 2047-2059, Sep. 2016.

HAWLITSCHEK, F.; TEUBNER, T.; WEINHARDT, C. Trust in the sharing economy: . Die Unternehmung- Swiss Journal of Business Research and Practice, [s. ], v. 70, n. 1, p. 26-44, Apr.2016.

HILL, J. A.; WELLMAN, Michael. Peer-to-Peer Tangible Goods Rental. In: Joint Workshop on Trading Agent Design and Analysis (TADA) and Agent-Mediated Electronic Commerce (AMEC), 1., 2011, Valencia, Spain. Anais [...].Valencia, Spain: AAMAS, 2011. p. 1-14.

HOU, L. Destructive Sharing Economy: a passage from Status to Contract. Computer Law \& Security Review, [s. ], v. 31, n.4, p.965-976, 2018.

HUNT, S. Marketing theory: foundations, controversy, strategy, and resource-advantage theory. New York: Routledge, 2010.

KANNISTO, P. Travelling like locals: market resistance in long-term travel. Tourism Management, [s. ], v. 67, p. 297-306, 2018.

KARLSSON, L.; KEMPERMAN, A. D. A. M.; DOLNICAR, S. May I sleep in your bed? Getting permission to book. Annals of Tourism Research, [s. I], v. 62, p. 1-12, Jan. 2017.

LAURELL, C.; SANDSTRÖM, C. Analysing Uber in social media-disruptive technology or institutional disruption?. International Journal of Innovation Management, [s. I, v. 20, n. 7, p. 1640013-1-19, 2016.

LIANG, S. et al. Be a "Superhost": the importance of badge systems for peer-to-peer rental accommodations. Tourism management, [s. ]], v. 60, p. 454-465, Jun. 2017.

LUTZ, C.; NEWLANDS, G. Consumer segmentation within the sharing economy: the case of Airbnb. Journal of Business Research, [s. I], v. 88, n. C, p. 187-196, 2018.

MAIR, J.; REISCHAUER, G. Capturing the dynamics of the sharing economy: institutional research on the plural forms and practices of sharing economy organizations. Technological Forecasting and Social Change, [s. I], v. 125, n. C, p. 11-20, 2017.

MOELLER, S.; WITTKOWSKI, K. The burdens of ownership: reasons for preferring renting. Managing Service Quality, [s. ]], v. 20, n. 2, p. 176-191, 2010.

MOLZ, J. Social networking technologies and the moral economy of alternative tourism: the case of couchsurfing. Org. Annals of Tourism Research, [s. I], v. 43, p. 210-230, Oct. 2013.

MUÑOZ, P.; COHEN, B. Mapping out the sharing economy: a configurational approach to sharing business modeling. Technological Forecasting and Social Change, [s. ], v. 125, p. 21-37, 2017.

OPPERMAN, A. Por que Uber e Airbnb deram certo, enquanto taxi magic e couchsurfing falharam. Época Negócios, São Paulo, maio 2017. Seção Carreira. Disponível em: https://epocanegocios.globo.com/Carreira/noticia/2017/05/ por-que-uber-e-airbnb-deram-certo-enquanto-taxi-magic-e-couchsurfing-falharam.html. Acesso em 29 maio 2018.

PIZAM, A. Peer-to-peer travel: blessing or blight? International Journal of Hospitality Management, [s. I], v. 38, p. 118-119, 2014.

PRICE WATER HOUSE INTERNATIONAL LTD. The Sharing Economy: consumer intelligence series. 2015. Disponível 
em: https://www.pwc.com/us/en/technology/publications/ assets/pwc-consumer-intelligence-series-the-sharing-economy. pdf. Acesso em: 29 de maio de 2018.

RAHDARI, A.; SEPASI, S.; MORADI, M. Achieving sustainability through Schumpeterian social entrepreneurship: the role of social enterprises. Journal of Cleaner Production, [s. I], v. 137, p. 347-360, 2016.

RÉVILLION, A. S. P. A utilização de pesquisas exploratórias na área de marketing. Revista Interdisciplinar de Marketing, Maringá, v. 2, n. 2, p. 21-37, 2015.

RIHOVA, I. et al. Customer-to-customer co-creation practices in tourism: lessons from Customer-Dominant logic. Tourism Management, [s. I], v. 67, p. 362-375, Aug. 2018.

SANTOS, J. V. T. dos. A construção da viagem inversa: ensaio sobre a investigação nas ciências sociais. Cadernos de Sociologia, Porto Alegre, v. 3, n. 3, p. 55-88, 1991.

SIGALA, M. Market formation in the sharing economy: findings and implications from the sub-economies of Airbnb. In: BARILE, S.; PELLICANO, M.; POLESE, F. (eds.) Social Dynamics in a Systems Perspective. [S.], Springer, 2018. Chapter 9, p. 159-174.

SO, K. K. F.; OH, H.; MIN, S. Motivations and constraints of Airbnb consumers: findings from a mixed-methods approach. Tourism Management, [s. I], v. 67, p. 224-236, Jan. 2018.

SOUZA, C. A. P de; LEMOS, R. Aspectos jurídicos da economia do compartilhamento: função social e tutela da confiança. Revista de Direito da Cidade, Rio de Janeiro, v. 8, n. 4, p. 1757- 1777, 2016.

TUSSYADIAH, I.; PARK, S. When guests trust hosts for their words: host description and trust in sharing economy. Tourism Management, [s. I], v. 67, p. 261-272, 2018.

TUSSYADIAH, I.; PESONEN, J. Impacts of peer-to-peer accommodation use on travel patterns. Journal of Travel Research, [s. I], v. 55, n. 8, p. 1022-1040, 2016.

WITTKOWSKI, K.; MOELLER, S.; WIRTZ, J. Firms' intentions to use non ownership services. Journal of Service Research, [s. I], v. 16, n. 2, p. 171-185, 2013.

WU, X.; ZHI, Q. Impact of shared economy on urban sustainability: from the perspective of social, economic, and environmental sustainability. Energy Procedia, [s. I], v. 104, p. 191-196, Dec. 2016.

XIANG, Z. From digitization to the age of acceleration: on information technology and tourism. Tourism Management Perspectives, [s. ]], v. 25, p 147-150, Dec. 2017.

YANG, Y.; TAN, K.; LI, X. Antecedents and consequences of home-sharing stays: evidence from a nationwide household tourism survey. Tourism Management, [s. ], v. 70, p. 15-28, Feb. 2019.

YE, S.; XIAO, H.; ZHOU, L. Commodification and perceived authenticity in commercial homes. Annals of Tourism Research, [s. I], v. 71, p. 39-53, Jul. 2018.

\section{Contato:}

Francis Marcean Resende Barros

E-mail: francis031995@gmail.com

Marlusa de Sevilha Gosling

E-mail: mg.ufmg@gmail.com 